Received: 15 May 2018

Accepted: 17 August 2018

Published online: 06 September 2018

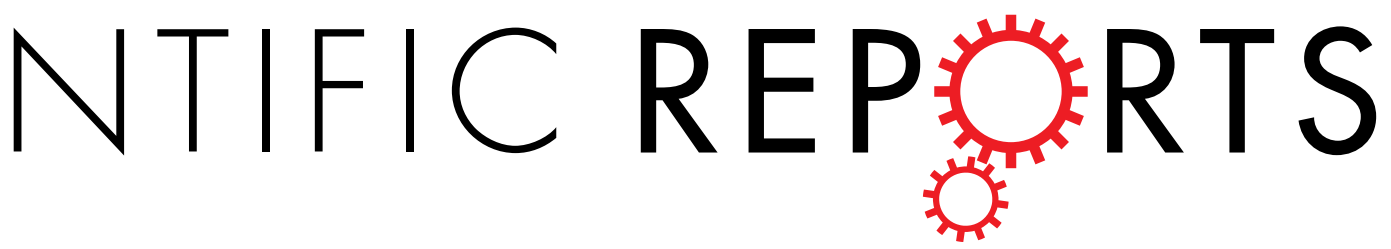

\title{
Low Gilbert Damping Constant in Perpendicularly Magnetized W/CoFeB/MgO Films with High Thermal Stability
}

\author{
Dustin M. Lattery $\mathbb{1}^{1}$, Delin Zhang ${ }^{2}$, Jie Zhu ${ }^{1}$, Xudong Hang $\mathbb{D}^{2}$, Jian-Ping Wang ${ }^{2}$ \& \\ Xiaojia Wang ${ }^{1,2}$
}

Perpendicular magnetic materials with low damping constant and high thermal stability have great potential for realizing high-density, non-volatile, and low-power consumption spintronic devices, which can sustain operation reliability for high processing temperatures. In this work, we study the Gilbert damping constant $(\alpha)$ of perpendicularly magnetized $\mathrm{W} / \mathrm{CoFeB} / \mathrm{MgO}$ films with a high perpendicular magnetic anisotropy (PMA) and superb thermal stability. The $\alpha$ of these PMA films annealed at different temperatures $\left(T_{\mathrm{ann}}\right)$ is determined via an all-optical Time-Resolved Magneto-Optical Kerr Effect method. We find that $\alpha$ of these W/CoFeB/MgO PMA films decreases with increasing $T_{\text {ann, }}$ reaches a minimum of $\alpha=0.015$ at $T_{\mathrm{ann}}=350^{\circ} \mathrm{C}$, and then increases to 0.020 after post-annealing at $400^{\circ} \mathrm{C}$. The minimum $\alpha$ observed at $350^{\circ} \mathrm{C}$ is rationalized by two competing effects as $T_{\text {ann }}$ becomes higher: the enhanced crystallization of $\mathrm{CoFeB}$ and dead-layer growth occurring at the two interfaces of the $\mathrm{CoFeB}$ layer. We further demonstrate that $\alpha$ of the $400^{\circ} \mathrm{C}$-annealed W/CoFeB/MgO film is comparable to that of a reference $\mathrm{Ta} / \mathrm{CoFeB} / \mathrm{MgO} \mathrm{PMA}$ film annealed at $300^{\circ} \mathrm{C}$, justifying the enhanced thermal stability of the W-seeded CoFeB films.

Since the first demonstration of perpendicular magnetic tunnel junctions with perpendicular magnetic anisotropy (PMA) $\mathrm{Ta} / \mathrm{CoFeB} / \mathrm{MgO}$ stacks ${ }^{1}$, interfacial PMA materials have been extensively studied as promising candidates for ultra-high-density and low-power consumption spintronic devices, including spin-transfer-torque magnetic random access memory (STT-MRAM) ${ }^{2,3}$, electrical-field induced magnetization switching ${ }^{4-6}$, and spin-orbit torque (SOT) devices ${ }^{7-9}$. An interfacial PMA stack typically consists of a thin ferromagnetic layer (e.g., $\mathrm{CoFeB}$ ) sandwiched between a heavy metal layer (e.g., Ta) and an oxide layer (e.g., MgO). The heavy metal layer interface with the ferromagnetic layer is responsible for the spin Hall effect, which is favorable for SOT and skyrmion devices ${ }^{10,11}$. The critical switching current $\left(J_{\mathrm{c} 0}\right)$ should be minimized to decrease the power consumption of perpendicular STT-MRAM and SOT devices. Reducing $J_{\mathrm{c} 0}$ requires the exploration of new materials with low Gilbert damping constant $(\alpha)$ and large spin Hall angle $\left(\theta_{\text {SHE }}\right)$. Meanwhile, a large effective anisotropy $\left(K_{\text {eff }}\right)$ is also favorable to maintain thermal stability ${ }^{12,13}$.

In addition, spintronic devices need to sustain operation reliability for processing temperatures as high as $400^{\circ} \mathrm{C}$ for their integration with existing CMOS fabrication technologies, providing the standard back-end-of-line process compatibility ${ }^{14}$. Based on this requirement, the magnetic properties of a PMA material should be thermally stable at annealing temperatures $\left(T_{\text {ann }}\right)$ up to $400^{\circ} \mathrm{C}$. Unfortunately, $\mathrm{Ta} / \mathrm{CoFeB} / \mathrm{MgO} \mathrm{PMA}$ films commonly used in spintronic devices cannot survive with $T_{\text {ann }}$ higher than $350^{\circ} \mathrm{C}$, due to Ta diffusion or CoFeB oxidation at the interfaces ${ }^{15-17}$. The diffusion of Ta atoms can act as scattering sites to increase the spin-flip probability ${ }^{18}$ and lead to a higher Gilbert Damping constant $(\alpha)$, a measure of the energy dissipation from the magnetic precession into phonons or magnons ${ }^{19}$.

Modifying the composition of thin-film stacks can prevent heavy metal diffusion, which is beneficial to both lowering $\alpha$ and improving thermal stability ${ }^{20}$. Along this line, new interfacial PMA stacks have been developed, such as

${ }^{1}$ Department of Mechanical Engineering, University of Minnesota, Minneapolis, MN, 55455, USA. ${ }^{2}$ Department of Electrical and Computer Engineering, University of Minnesota, Minneapolis, MN, 55455, USA. Dustin M. Lattery and Delin Zhang contributed equally. Correspondence and requests for materials should be addressed to J.-P.W. (email: jpwang@umn.edu) orX.W. (email: wang4940@umn.edu) 
$\mathrm{Mo} / \mathrm{CoFeB} / \mathrm{MgO}$, to circumvent the limitation on device processing temperatures ${ }^{21,22}$. While $\mathrm{Mo} / \mathrm{CoFeB} / \mathrm{MgO}$ films can indeed exhibit PMA at temperatures higher than $400^{\circ} \mathrm{C}$, they cannot be used for SOT devices due to the weak spin Hall effect of the Mo layer ${ }^{21,22}$. Recently, W/CoFeB/MgO PMA thin films have been proposed because of their PMA property at high post-annealing temperature ${ }^{23}$, and the large (negative) spin Hall angle of the W layer $\left(\theta_{\mathrm{SHE}} \approx\right.$ $-0.30)^{24}$, which is twice that of a Ta layer $\left(\theta_{\mathrm{SHE}} \approx-0.12 \sim-0.15\right)^{9,25}$. While there have been a few scattered studies demonstrating the promise of fabricating SOT devices using the $\mathrm{W} / \mathrm{CoFeB} / \mathrm{MgO}$ stacks, special attention has been given to their PMA properties and functionalities as SOT devices ${ }^{26,27}$, or the damping of in-plane W/CoFeB stacks ${ }^{28}$. A systematic investigation is lacking on the effect of $T_{\text {ann }}$ on $\alpha$ of W/CoFeB/MgO PMA thin films with perpendicular anisotropy, as well as the physical mechanisms that alter $\alpha$ after post-annealing.

\section{Methods}

Sample preparation and magnetic characterization. In this work, we grow a series of W(7)/ $\mathrm{Co}_{20} \mathrm{Fe}_{60} \mathrm{~B}_{20}(1.2) / \mathrm{MgO}(2) / \mathrm{Ta}(3)$ thin films on $\mathrm{Si} / \mathrm{SiO}_{2}(300)$ substrates (thickness in nanometers) with a magnetron sputtering system $\left(<5 \times 10^{-8}\right.$ Torr $)$. These films are post-annealed at varying temperatures $\left(T_{\text {ann }}=250-350^{\circ} \mathrm{C}\right.$ for 1 hour, $400^{\circ} \mathrm{C}$ for 30 minutes) within a high-vacuum furnace $\left(<1 \times 10^{-6} \mathrm{Torr}\right)$. After post-annealing, the magnetic properties and damping constants of these films are systematically investigated as a function of $T_{\text {ann }}$. For comparison, a reference sample of $\mathrm{Ta}(7) / \mathrm{Co}_{20} \mathrm{Fe}_{60} \mathrm{~B}_{20}(1.2) / \mathrm{MgO}(2) / \mathrm{Ta}(3)$ is also prepared to examine the effect of seeding layer to the damping constant of these PMA films. The effective saturation magnetization $\left(M_{\mathrm{s}}\right)$ and anisotropy of these films are measured with the Vibrating Sample Magnetometer (VSM) module of a Physical Property Measurement System (Quantum Design, DynaCool).

TR-MOKE measurements and data reduction. The magnetization dynamics of these PMA thin films are determined using the all-optical Time-Resolved Magneto-Optical Kerr Effect (TR-MOKE) method ${ }^{29-34}$. This pump-probe method utilizes ultra-short laser pulses to thermally demagnetize the sample and probe the resulting Kerr rotation angle $\left(\theta_{\mathrm{K}}\right)$. In the polar-MOKE configuration, $\theta_{\mathrm{K}}$ is proportional to the change of the out-of-plane component of magnetization ${ }^{35}$. Details of the TR-MOKE setup are provided in Section S2 of the SI.

The TR-MOKE signal is fitted to the equation

$$
\theta_{\mathrm{K}}=A+B e^{-t / C}+D \sin (2 \pi f t+\varphi) e^{-t / \tau},
$$

where $A, B$, and $C$ are the offset, amplitude, and exponential decaying constant of the thermal background, respectively. $D$ denotes the amplitude of oscillations, $f$ is the resonance frequency, $\varphi$ is a phase shift (related to the demagnetization process), and $\tau$ is the relaxation time of magnetization precession. Directly from TR-MOKE measurements, an effective damping constant $\left(\alpha_{\text {eff }}\right)$ can be extracted based on the relationship $\alpha_{\text {eff }}=1 /(2 \pi f \tau)$. However, $\alpha_{\text {eff }}$ is not an intrinsic material property; rather, it depends on measurement conditions, such as the applied field direction $\left(\theta_{\mathrm{H}}\right)$, the magnitude of the applied field $\left(H_{\mathrm{ext}}\right)$, and inhomogeneities of the sample (e.g. local variation in the magnetic properties of the sample) $)^{36,37}$.

To obtain the Gilbert damping constant, the inhomogeneous contribution needs to be removed from $\alpha_{\text {eff }}$, such that the remaining value of damping is an intrinsic material property and independent of the measurement conditions. To determine the inhomogeneous broadening in the sample, the effective anisotropy field $\left(H_{k \text {,eff }}=2 K_{\text {eff }} / M_{\mathrm{s}}\right)$ needs to be pre-determined from either (1) the magnetic hysteresis loops; or (2) the fitting results of $f$ vs. $H_{\text {ext }}$ obtained from TR-MOKE. The resonance frequency, $f$, can be related to $H_{\text {ext }}$ through the Smit-Suhl approach by identifying the second derivatives of the total magnetic free energy, which combines a Zeeman energy, an anisotropy energy, and a demagnetization energy ${ }^{38-40}$. For a perpendicularly magnetized thin film, $f$ is defined by Eqs $1-4^{40}$.

$$
\begin{gathered}
f=\frac{\gamma}{2 \pi} \sqrt{H_{1} H_{2}}, \\
H_{1}=H_{\text {ext }} \cos \left(\theta-\theta_{\mathrm{H}}\right)+H_{\mathrm{k}, \mathrm{eff}} \cos ^{2}(\theta), \\
H_{2}=H_{\mathrm{ext}} \cos \left(\theta-\theta_{\mathrm{H}}\right)+H_{\mathrm{k}, \mathrm{eff}} \cos (2 \theta), \\
2 H_{\mathrm{ext}} \sin \left(\theta_{\mathrm{H}}-\theta\right)=H_{\mathrm{k}, \mathrm{eff}} \sin (2 \theta) .
\end{gathered}
$$

This set of equations permits calculation of $f$ with the material gyromagnetic ratio $(\gamma), H_{\mathrm{ext}}, \theta_{\mathrm{H}}, H_{\mathrm{k}, \mathrm{eff}}$, and the angle between the equilibrium magnetization direction and the surface normal ( $\theta$, determined by Eq. 4$)$. The measured values of $f$ as a function of $H_{\text {ext }}$ can be fitted to Eq. 1 by treating $\gamma$ and $H_{\mathrm{k} \text {,eff }}$ as fitting parameters. To minimize the fitting errors resulting from the inhomogeneous broadening effect that is pronounced at the low fields, we use measured frequencies at high fields $\left(H_{\text {ext }}>10 \mathrm{kOe}\right)$ to determine $H_{\mathrm{k}, \mathrm{eff}}$.

With a known value of $H_{\mathrm{k}, \mathrm{eff}}$, the Gilbert damping constant of the sample can be determined through a fitting of the inverse relaxation time $(1 / \tau)$ to Eq. 5. The two terms of Eq. 5 take into account, respectively, contributions from the intrinsic Gilbert damping of the materials (first term) and inhomogeneous broadening (second term) ${ }^{36}$ :

$$
\frac{1}{\tau}=\frac{1}{2} \alpha \gamma\left(H_{1}+H_{2}\right)+\frac{1}{2}\left|\frac{d \omega}{d H_{\mathrm{k}, \mathrm{eff}}}\right| \Delta H_{\mathrm{k}, \mathrm{eff}},
$$

where $H_{1}$ and $H_{2}$ are related to the curvature of the magnetic free energy surface as defined by Eqs 2 and $3^{40,41}$. The second term on the right side of Eq. 5 captures the inhomogeneous effect by attributing it to a spatial variation 

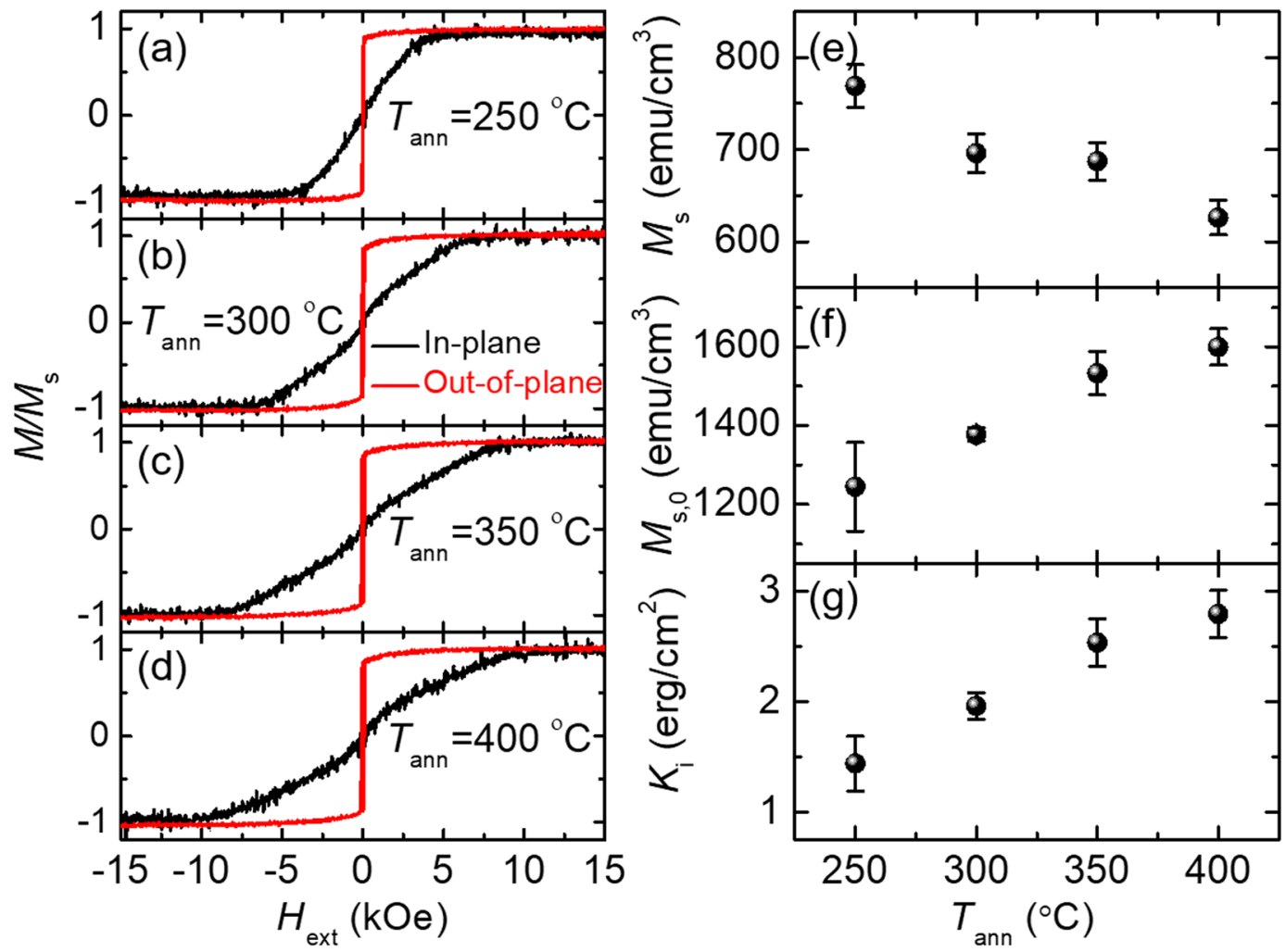

Figure 1. Room temperature magnetic hysteresis loops of $\mathrm{W} / \mathrm{CoFeB} / \mathrm{MgO} \mathrm{PMA}$ thin films post-annealed at (a) $250^{\circ} \mathrm{C}$, (b) $300^{\circ} \mathrm{C}$, (c) $350^{\circ} \mathrm{C}$, and (d) $400^{\circ} \mathrm{C}$. Black and red curves denote external magnetic field $\left(H_{\text {ext }}\right)$ applied along and perpendicular to the film plane, respectively. (e-g) Plots of the effective saturation magnetization $\left(M_{\mathrm{s}}\right)$, the intrinsic saturation magnetization (i.e., excluding the effect of the dead layer, $M_{\mathrm{s}, 0}$ ), and the interfacial anisotropy $\left(K_{\mathrm{i}}\right)$ as functions of $T_{\mathrm{ann}}$.

in the magnetic properties $\left(\Delta H_{\mathrm{k}, \text { eff }}\right)$, analogous to the linewidth broadening effect in Ferromagnetic Resonance measurements ${ }^{42}$. The magnitude of $d \omega / d H_{\mathrm{k}, \text { eff }}$ can be calculated once the relationship of $\omega$ vs. $H_{\text {ext }}$ is determined with a numerical method. Both $\alpha$ and $\Delta H_{\mathrm{k} \text {,eff }}$ (the inhomogeneous term related to the amount of spatial variation in $\left.H_{\mathrm{k}, \mathrm{eff}}\right)$ are determined via the fitting of the measured $1 / \tau$ based on Eq. 5 . In this way, we can uniquely extract the field-independent $\alpha$, as an intrinsic material property, from the effective damping $\left(\alpha_{\text {eff }}\right)$, which is directly obtained from TR-MOKE and dependent on $H_{\text {ext }}$.

It should be noted here that the inhomogeneous broadening of the magnetization precession is presumably due to the multi-domain structure of the materials, which becomes negligible in the high-field regime $\left(H_{\text {ext }} \gg H_{\mathrm{k}, \text { eff }}\right)$ as the magnetization direction of multiple magnetic domains becomes uniform. This is also reflected by the fact that the derivative in the second term of Eq. 5 approaches zero for the high-field regime ${ }^{43}$.

\section{Results and Discussion}

Figure 1 plots the magnetic hysteresis loops and associated magnetic properties extracted from VSM measurements. With the increase of $T_{\text {ann }}, M_{\mathrm{s}}$ for the $\mathrm{W} / \mathrm{CoFeB} / \mathrm{MgO}$ films decreases from $\sim 780$ to $\sim 630 \mathrm{emu} \mathrm{cm}^{-3}$ (Fig. 1e). The PMA in the $\mathrm{W} / \mathrm{CoFeB} / \mathrm{MgO}$ films is dominated by the interface anisotropy $\left(K_{\mathrm{i}}\right)$, which increases from 1.4 to $2.8 \mathrm{erg} \mathrm{cm}^{-2}$ (excluding the dead-layer thickness effect) with $T_{\text {ann }}$ up to $400^{\circ} \mathrm{C}$ (Fig. $1 \mathrm{~g}$ ). If the film thicknesses are corrected by subtracting the dead layer, $K_{\mathrm{i}}$ will change from 1.3 to $1.6 \mathrm{erg} \mathrm{cm}{ }^{-2}$ as $T_{\text {ann }}$ increases from 250 to $400^{\circ} \mathrm{C}$, which agrees better with literature values ${ }^{44}$. Details about the determination of $K_{\mathrm{i}}$ including the dead-layer effect are provided in Section S1 of the Supplementary Information (SI).

We attribute the decrease of $M_{\mathrm{s}}$ at high $T_{\text {ann }}$ to the growth of a dead layer at the CoFeB interfaces, which becomes prominent at higher $T_{\mathrm{ann}}$. To quantitatively determine the thickness of the dead layer as $T_{\text {ann }}$ increases, we prepare four sets of PMA stacks of $\mathrm{W}(7) / \mathrm{CoFeB}(t) / \mathrm{MgO}(2) / \mathrm{Ta}(3)$. One set contains five stacks with varying thicknesses for the CoFeB layer $(t=1.2,1.5,1.8,2.2$, and $2.5 \mathrm{~nm})$ and is post-annealed at a fixed $T_{\text {ann }}$. Four $T_{\text {ann }}$ of $250,300,350$, and $400^{\circ} \mathrm{C}$ are used for four sets of the PMA stacks, respectively. The annealing conditions are the same as those for the $\mathrm{W}(7) / \mathrm{CoFeB}(1.2) / \mathrm{MgO}(2) / \mathrm{Ta}(3)$ samples discussed previously. We measure the magnetic hysteresis loops of these samples using VSM and plot their saturation magnetization area product $\left(M_{\mathrm{S}} \times t\right)$ as a function of film thickness $(t)$ in Fig. 2. Linear extrapolation of the $M_{\mathrm{S}} \times t$ data provides the dead-layer thickness, at which the magnetization reduces to zero as illustrated by the $x$-axis intercept in Fig. 2 . The slope of the linear fit also provides intrinsic saturation magnetization $\left(M_{\mathrm{s}, 0}\right)$, which corresponds to the saturation magnetization after the removal of the dead-layer effect. The values of $M_{\mathrm{s} 0}$ (Fig. 1f) show an increasing trend with $T_{\text {ann }}$ from $\sim 1300$ to $\sim 1600 \mathrm{emu} \mathrm{cm}^{-3}$, which agrees well with previous measurement results for $\mathrm{W} / \mathrm{CoFeB} / \mathrm{MgO}$ films ${ }^{44}$. 


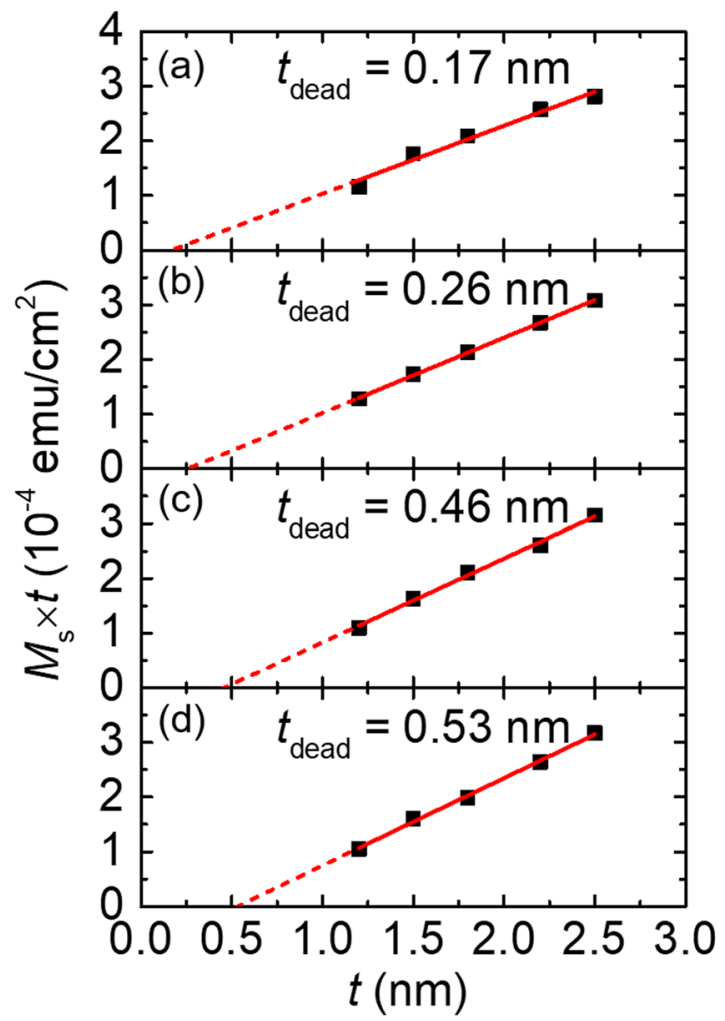

Figure 2. The dead-layer extraction results. (a-d) represent the series of samples annealed at 250, 300, 350, and $40{ }^{\circ} \mathrm{C}$ respectively. The $t_{\text {dead }}$ value is the extrapolated $x$-axis intercept from the linear fitting of the thicknessdependent saturation magnetization area product $\left(M_{\mathrm{s}} \times t\right)$.

Figure 3a depicts the schematic of polar TR-MOKE experiments with the definition of several parameters and angles that are important for the data reduction (Section 2.2). Figure $3 \mathrm{~b}$ plots the TR-MOKE signals (symbols) and the model fitting of $\theta_{\mathrm{K}}$ (black lines) as functions of time delay for the $400^{\circ} \mathrm{C} \mathrm{W} / \mathrm{CoFeB} / \mathrm{MgO}$ sample at an external field angle of $\theta_{\mathrm{H}}=76^{\circ}$. To determine the values of $H_{\mathrm{k}, \text { eff }}$ and $\alpha$, the TR-MOKE measurements are conducted at varying $H_{\text {ext }}$ from 2 to $20 \mathrm{kOe}$. Both the $f$ and $\tau$ of the measured oscillations, resulting from the magnetization precession, depend greatly on $H_{\text {ext }}$ as predicted by Eqs 1 and 5 .

By repeating this measurement at varying $\theta_{\mathrm{H}}$, we can show that $\alpha$ is an intrinsic material property, independent of $\theta_{\mathrm{H}}$. Figure 4 a plots the resonance frequencies derived from TR-MOKE and model fittings for the $400^{\circ} \mathrm{C}$ sample at two field directions $\left(\theta_{\mathrm{H}}=76^{\circ}\right.$ and $\left.89^{\circ}\right)$. For the data acquired at $\theta_{\mathrm{H}}=89^{\circ}$, a minimum $f$ occurs at $H_{\mathrm{ext}} \approx$ $H_{\mathrm{k}, \mathrm{eff}}$. This corresponds to the smallest amplitude of magnetization precession, when the equilibrium direction of the magnetization is aligned with the applied field direction at the magnitude of $H_{\mathrm{k}, \mathrm{eff}}{ }^{40}$. The dip at this local minimum diminishes when $\theta_{\mathrm{H}}$ decreases, as reflected by the comparison between the red $\left(\theta_{\mathrm{H}}=89^{\circ}\right)$ and blue $\left(\theta_{\mathrm{H}}=76^{\circ}\right)$ lines in Fig. $4 \mathrm{a}$. With the $H_{\mathrm{k}, \text { eff }}$ extracted from the fitting of frequency data with $\theta_{\mathrm{H}}=89^{\circ}$, we generate the plot of theoretically predicted $f$ vs. $H_{\text {ext }}\left(\theta_{\mathrm{H}}=76^{\circ}\right.$ theory, blue line in Fig. $\left.4 \mathrm{a}\right)$, which agrees well with experimental data (open squares in Fig. 4a).

The inverse relaxation time $(1 / \tau)$ should also have a minimum value near $H_{\mathrm{k}, \mathrm{eff}}$ for $\theta_{\mathrm{H}}=89^{\circ}$ if the damping was purely from Gilbert damping (as shown by the solid lines in Fig. 4b,d); however, the measured data do not follow this trend. Adding the inhomogeneous term (dotted lines in Fig. 4b,d) more accurately describes the field dependence of the measured $1 / \tau$ (open symbols in Fig. $4 \mathrm{~b}, \mathrm{~d}$ ) It should be noted that the dip of the predicted $1 / \tau$ occurs when the frequency derivative term in Eq. 5 approaches zero; however, this is not captured by the measurement. Figure $4 \mathrm{c}$,e depict the field-dependent effective damping $\left(\alpha_{\text {eff }}\right)$ calculated using the Gilbert damping $(\alpha)$ extracted from fitting the measured $1 / \tau$.

With the knowledge that the value of $\alpha$ extracted with this method is the intrinsic material property, we repeat this data reduction technique for the annealed $\mathrm{W} / \mathrm{CoFeB} / \mathrm{MgO}$ samples discussed in Fig. 1. The symbols in Fig. 5 represent the resonance frequencies and damping constants (both effective damping and Gilbert damping) for all samples measured at $\theta_{\mathrm{H}} \approx 90^{\circ}$. The fittings for the resonance frequency based on Eq. 1 (red lines) are also shown to demonstrate the good agreement between our TR-MOKE measurement and theoretical prediction. The uncertainties of $f, \tau$, and $H_{\mathrm{k}, \text { eff }}$ are calculated from the least-squares fitting uncertainty and the uncertainty of measuring $H_{\text {ext }}$ with the Hall sensor.

The summary of the anisotropy and damping measured via TR-MOKE is shown in Fig. 6. Figure 6a plots $H_{\mathrm{k}, \text { eff }}$ obtained from VSM (black open circles) and TR-MOKE (blue open squares), both of which exhibit a monotonic increasing trend as $T_{\text {ann }}$ becomes higher. Discrepancies in $H_{\mathrm{k}, \text { eff }}$ from these two methods can be attributed to the difference in the size of the probing region, which is highly localized in TR-MOKE but sample-averaged in VSM. 

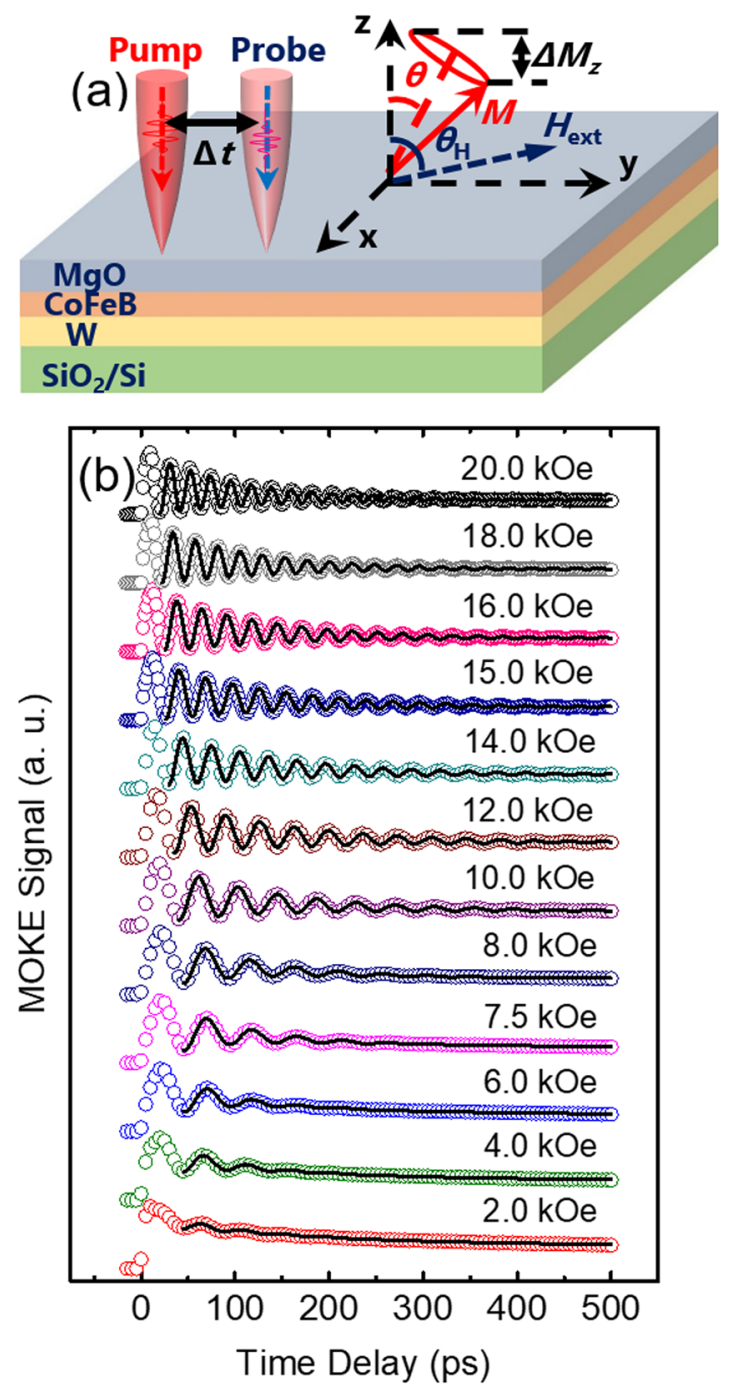

Figure 3. (a) Definition of the parameters and angles used in TR-MOKE experiments. The red circle indicates the magnetization precession. $\theta$ is the equilibrium direction of the magnetization. $\theta_{\mathrm{K}}$ is measured by the probe beam at a given time delay $(\Delta t)$. (b) The TR-MOKE data (open symbols) and model fitting of $\theta_{\mathrm{K}}$ (black curves) for the $400^{\circ} \mathrm{C}$ sample at $76^{\circ}$, for varying $H_{\text {ext }}$ from 2.0 to $20 \mathrm{kOe}$.

Since $H_{\mathrm{k}, \mathrm{eff}}$ determined from TR-MOKE is obtained from fitting the measured frequency for a localized region, we expect these values more consistently describe the magnetization precession than those obtained from VSM. The increase in $H_{\mathrm{k}, \text { eff }}$ with $T_{\mathrm{ann}}$ has previously be partially attributed to the crystallization of the CoFeB layer ${ }^{37}$. For temperatures higher than $350^{\circ} \mathrm{C}$, this increasing trend of $H_{\mathrm{k}, \text { eff }}$ begins to lessen, presumably due to the diffusion of $\mathrm{W}$ atoms into the $\mathrm{CoFeB}$ layer, which is more pronounced at higher $T_{\mathrm{ann}}$. The $\mathrm{W}$ diffusion process is also responsible for the decrease in $M_{\mathrm{s}}$ of the CoFeB layer as $T_{\text {ann }}$ increases (Fig. 1e). Subsequently, the decrease in $M_{\mathrm{s}}$ leads to a further-reduced demagnetizing energy and thus a larger $H_{\mathrm{k}, \text { eff. }}$.

Similar observation of $M_{s}$ has been reported in literature for Ta/CoFeB/MgO PMA structures and attributed to the growth of a dead layer at the heavy metal/CoFeB interface ${ }^{1}$. Figure $6 \mathrm{~b}$ summarizes $t_{\text {dead }}$ as a function of $T_{\text {ann }}$ with $t_{\text {dead }}$ increasing from 0.17 to $0.53 \mathrm{~nm}$ as $T_{\text {ann }}$ changes from 250 to $400{ }^{\circ} \mathrm{C}$, as discussed in Section II.

Figure $6 \mathrm{c}$ depicts the dependence of $\alpha$ on $T_{\text {ann }}$, which first decreases with $T_{\text {ann }}$, reaches a minimum of 0.015 at $350^{\circ} \mathrm{C}$, and then increases as $T_{\text {ann }}$ rises to $400^{\circ} \mathrm{C}$. Similar trends have been observed for $\mathrm{Ta} / \mathrm{CoFeB} / \mathrm{MgO}$ previously (minimum $\alpha$ at $\left.T_{\text {ann }}=300^{\circ} \mathrm{C}\right)^{37}$. We speculate that this dependence of damping on $T_{\text {ann }}$ is due to two competing effects: (1) the increase in crystallization in the CoFeB layer with $T_{\text {ann }}$ which reduces the damping, and (2) the growth of a dead layer, which results from the diffusion of $\mathrm{W}$ and $\mathrm{B}$ atoms and is prominent at higher $T_{\mathrm{ann}}$.

As the amorphous as-deposited $\mathrm{CoFeB}$ film begins to form ordered phases at elevated temperatures, the number of scattering sites in the film tend to decrease ${ }^{45,46}$. The increase in crystallinity of the W/CoFeB/MgO film with $T_{\mathrm{ann}}$ is demonstrated by the XRD analysis detailed in Section S5 of the SI. At $T_{\mathrm{ann}}=400^{\circ} \mathrm{C}$, the dead-layer formation leads to a larger damping presumably due to an increase in scattering sites (diffused $\mathrm{W}$ atoms) that contribute to spin-flip events, as described by the Elliot-Yafet relaxation mechanisms ${ }^{18}$. Additionally, $\mathrm{W}$ atoms can increase the spin-orbit coupling and thus the damping as the inter-diffusion increases with $T_{\text {ann }}{ }^{47}$. The observation that 

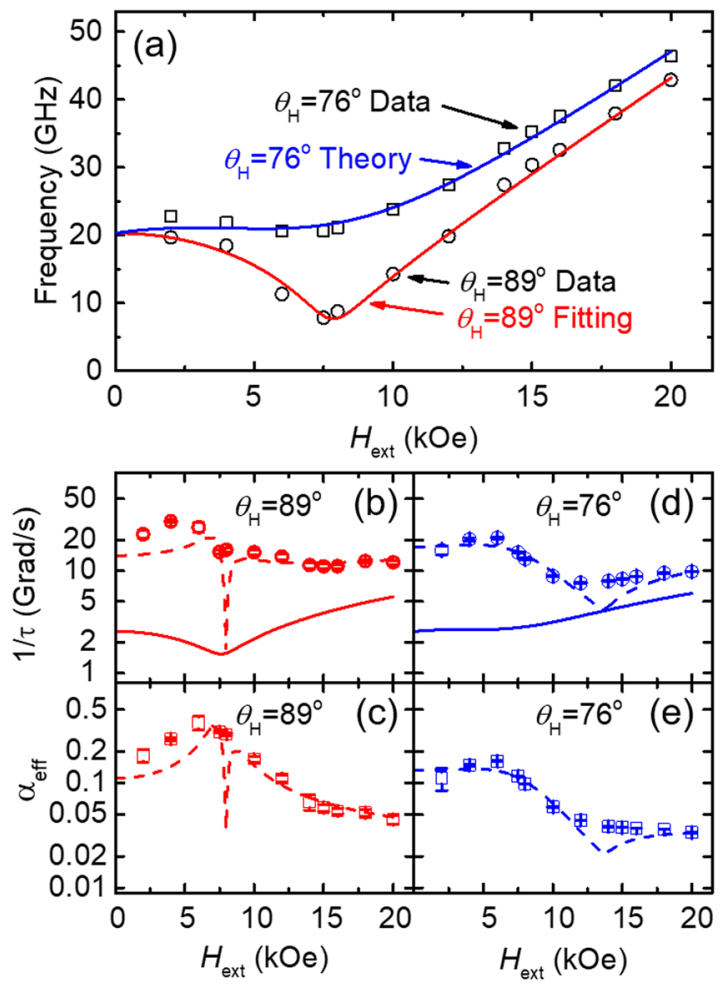

Figure 4. (a) Measured $f$ vs. $H_{\text {ext }}$ results for the $400^{\circ} \mathrm{C}$ sample at $\theta_{\mathrm{H}}=89^{\circ}$ (open circles) and $\theta_{\mathrm{H}}=76^{\circ}$ (open squares) and corresponding modeling at $\theta_{\mathrm{H}}=89^{\circ}$ (red line) and $\theta_{\mathrm{H}}=76^{\circ}$ (blue line). (b) The measured inverse of relaxation time $(1 / \tau)$ at $\theta_{\mathrm{H}}=89^{\circ}$ (open symbols) and the fitting of $1 / \tau$ based on Eq. 5 (dotted line). For reference, the first term of $1 / \tau$ in Eq. 5 is also plotted (solid line), which accounts for the contribution from the Gilbert damping only. (c) $\alpha_{\text {eff }}$ as a function of $H_{\mathrm{ext}}$ for $\theta_{\mathrm{H}}=89^{\circ}$ (red circles). The dotted line shows the predicted $\alpha_{\text {eff }}$ using the $\alpha$ extracted from the fitting of $1 / \tau$. (d,e) depict similar plots of $1 / \tau$ and $\alpha_{\text {eff }}$ for $\theta_{\mathrm{H}}=76^{\circ}$.

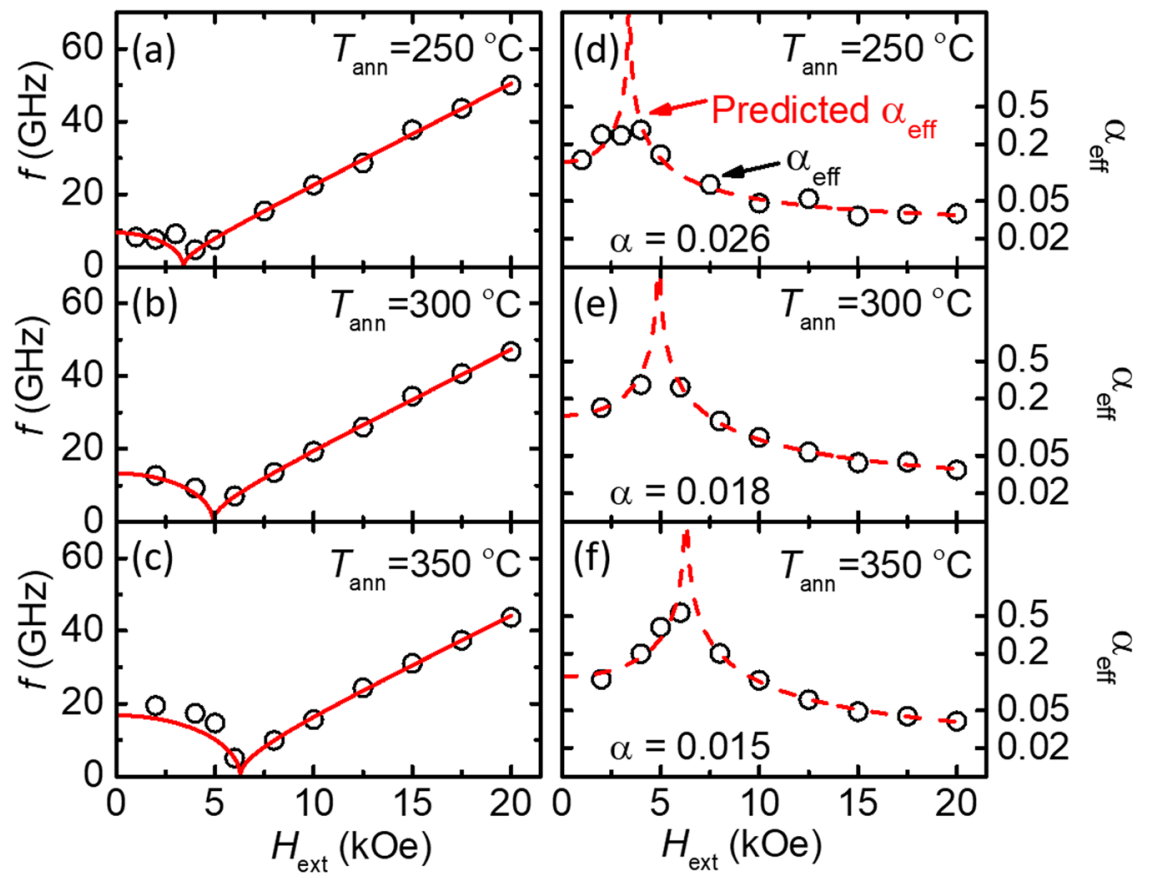

Figure 5. Results for $f(\mathbf{a}-\mathbf{c})$ and $\alpha_{\text {eff }}(\mathbf{d}-\mathbf{f})$, on a log scale, for individual samples (excluding the $400^{\circ} \mathrm{C}$ sample, which is discussed in Fig. 4). The fitting for $f$ is shown as a solid red line. The dashed line in (d-f) indicates the predicted $\alpha_{\text {eff }}$ from the values of $\alpha$ extracted from fitting $1 / \tau$. All three samples are measured at $\theta_{\mathrm{H}}=90^{\circ}$. 


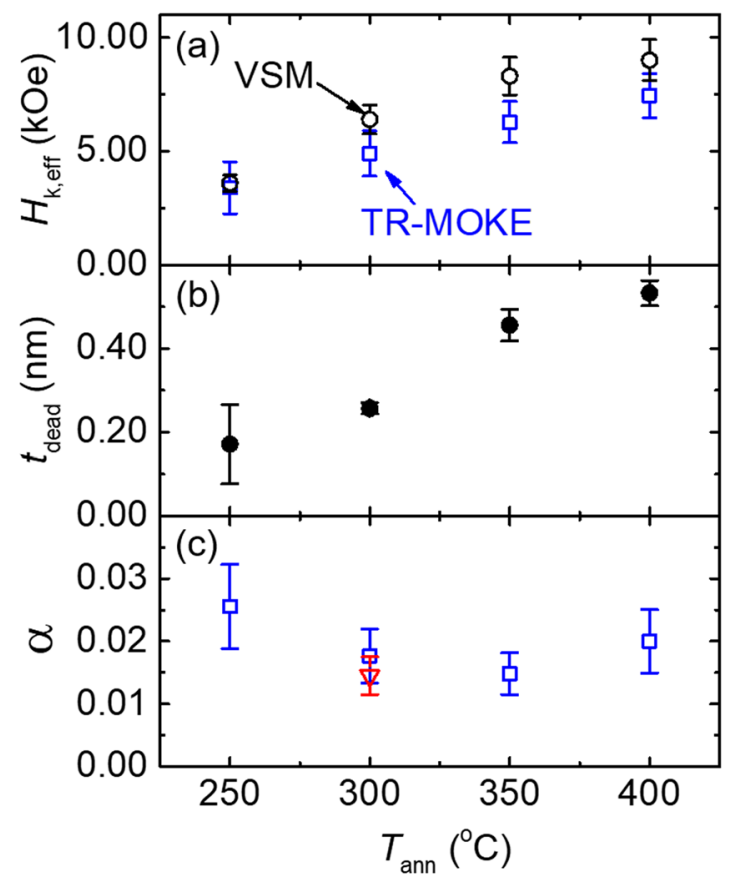

Figure 6. Summary of the magnetic properties of $\mathrm{W}$-seeded $\mathrm{CoFeB}$ as a function of $T_{\text {ann. }}$. (a) The dependence of $H_{\mathrm{k}, \mathrm{eff}}$ on $T_{\mathrm{ann}}$ obtained from both the VSM (black open circles) and TR-MOKE fitting (blue open squares). (b) The dependence of dead-layer thickness on $T_{\text {ann. }}$. Error bars are from standard error from a linear fit. (c) Damping constants as a function of $T_{\text {ann. }}$. The minimum damping constant of $\alpha=0.015$ occurs at $350^{\circ} \mathrm{C}$. The values for the all samples are obtained from measurements at $\theta_{\mathrm{H}} \approx 90^{\circ}$. For comparison, $\alpha$ of the reference Ta/ $\mathrm{CoFeB} / \mathrm{MgO}$ PMA sample annealed at $300^{\circ} \mathrm{C}$ is also shown as a red triangle in Fig. $6 \mathrm{c}$.

our W-seeded samples still sustain excellent PMA properties at $T_{\text {ann }}=400^{\circ} \mathrm{C}$ confirms their enhanced thermal stability, compared with $\mathrm{Ta} / \mathrm{CoFeB} / \mathrm{MgO}$ stacks which fail at $T_{\mathrm{ann}}=350^{\circ} \mathrm{C}$ or higher.

While the damping constants are comparable for the $\mathrm{W} / \mathrm{CoFeB} / \mathrm{MgO}$ and $\mathrm{Ta} / \mathrm{CoFeB} / \mathrm{MgO}$ films annealed at $300^{\circ} \mathrm{C}$, our work focuses on the enhanced thermal stability of W-seeded CoFeB PMA films that can maintain a relatively low damping constant $\left(0.020\right.$ at $\left.400^{\circ} \mathrm{C}\right)$. Such an advantage enables $\mathrm{W}$-seeded CoFeB layers to be viable and promising alternatives to $\mathrm{Ta} / \mathrm{CoFeB} / \mathrm{MgO}$, which is currently widely used in spintronic devices.

\section{Conclusion}

In summary, we deposit a series of W-seeded CoFeB PMA films with varying annealing temperatures up to $400^{\circ} \mathrm{C}$ and conduct ultrafast all-optical TR-MOKE measurements to study their magnetization precession dynamics. The Gilbert damping, as an intrinsic material property, is proven to be independent of measurement conditions, such as the amplitudes and directions of the applied field. The damping constant varies with $T_{\text {ann }}$, first decreasing and then increasing, leading to a minimum of $\alpha=0.015$ for the sample annealed at $350^{\circ} \mathrm{C}$. Due to the dead-layer growth, the damping constant slightly increases to $\alpha=0.020$ at $T_{\text {ann }}=400^{\circ} \mathrm{C}$, which demonstrates the improved enhanced thermal stability of $\mathrm{W} / \mathrm{CoFeB} / \mathrm{MgO}$ over the $\mathrm{Ta} / \mathrm{CoFeB} / \mathrm{MgO}$ structures. This strongly suggests the great potential of $\mathrm{W} / \mathrm{CoFeB} / \mathrm{MgO} \mathrm{PMA}$ systems for future spintronic device integration that requires materials to sustain a processing temperature as high as $400^{\circ} \mathrm{C}$.

\section{References}

1. Ikeda, S. et al. A perpendicular-anisotropy CoFeB-MgO magnetic tunnel junction. Nat. Mater. 9, 721-724 (2010).

2. Sato, $\mathrm{H}$. et al. Properties of magnetic tunnel junctions with a $\mathrm{MgO} / \mathrm{CoFeB} / \mathrm{Ta} / \mathrm{CoFeB} / \mathrm{MgO}$ recording structure down to junction diameter of $11 \mathrm{~nm}$. Appl. Phys. Lett. 105, 062403 (2014).

3. Sun, J. Z. et al. Spin-torque switching efficiency in CoFeB-MgO based tunnel junctions. Phys. Rev. B 88, 104426 (2013).

4. Zhu, J. et al. Voltage-induced ferromagnetic resonance in magnetic tunnel junctions. Phys. Rev. Lett. 108, 197203 (2012).

5. Wang, W. G., Li, M. G., Hageman, S. \& Chien, C. L. Electric-field-assisted switching in magnetic tunnel junctions. Nat. Mater. 11, 64-68 (2012).

6. Kanai, S. et al. Electric field-induced magnetization reversal in a perpendicular-anisotropy CoFeB-MgO magnetic tunnel junction. Appl. Phys. Lett. 101, 122403 (2012).

7. Yu, G. Q. et al. Switching of perpendicular magnetization by spin-orbit torques in the absence of external magnetic fields. Nat. Nanotechnol. 9, 548-554 (2014).

8. Bhowmik, D., You, L. \& Salahuddin, S. Spin Hall effect clocking of nanomagnetic logic without a magnetic field. Nat. Nanotechnol. 9, 59-63 (2014)

9. Liu, L. Q. et al. Spin-torque switching with the giant spin Hall effect of tantalum. Science 336, 555-558 (2012).

10. Jiang, W. J. et al. Blowing magnetic skyrmion bubbles. Science 349, 283-286 (2015).

11. Yu, G. et al. Room-temperature skyrmion shift device for memory application. Nano Lett. 17, 261-268 (2017).

12. Diao, Z. T. et al. Spin-transfer torque switching in magnetic tunnel junctions and spin-transfer torque random access memory. J. Phys.: Condens. Mat. 19, 165209 (2007). 
13. Manipatruni, S., Nikonov, D. E. \& Young, I. A. Energy-delay performance of giant spin Hall effect switching for dense magnetic memory. Appl. Phys. Express 7, 103001 (2014).

14. Annunziata, A. J. et al. Materials investigation for thermally-assisted magnetic random access memory robust against $400{ }^{\circ} \mathrm{C}$ temperatures. J. Appl. Phys. 117, 17B739 (2015).

15. Miyakawa, N., Worledge, D. C. \& Kita, K. Impact of Ta diffusion on the perpendicular magnetic anisotropy of Ta/CoFeB/MgO. IEEE Magn. Lett. 4, 1000104 (2013).

16. Yang, S. et al. Thermally stable perpendicular magnetic anisotropy features of $\mathrm{Ta} / \mathrm{TaO}{ }_{x} / \mathrm{Ta} / \mathrm{CoFeB} / \mathrm{MgO} / \mathrm{W}$ stacks via $\mathrm{TaO}_{x}$ underlayer insertion. J. Appl. Phys. 116, 113902 (2014).

17. Gan, H. D. et al. Origin of the collapse of tunnel magnetoresistance at high annealing temperature in $\mathrm{CoFeB} / \mathrm{MgO}$ perpendicular magnetic tunnel junctions. Appl. Phys. Lett. 99, 252507 (2011).

18. Elliott, R. J. Theory of the effect of spin-orbit coupling on magnetic resonance in some semiconductors. Phys. Rev. 96, 266-279 (1954).

19. Suhl, H. Theory of the magnetic damping constant. IEEE Trans. Magn. 34, 1834-1838 (1998).

20. Ikeda, S. et al. Tunnel magnetoresistance of $604 \%$ at $300 \mathrm{~K}$ by suppression of Ta diffusion in CoFeB/MgO/CoFeB pseudo-spin-valves annealed at high temperature. Appl. Phys. Lett. 93, 082508 (2008).

21. Liu, T., Zhang, Y., Cai, J. W. \& Pan, H. Y. Thermally robust Mo/CoFeB/MgO trilayers with strong perpendicular magnetic anisotropy. Sci. Rep. 4, 5895 (2014).

22. Almasi, H. et al. Enhanced tunneling magnetoresistance and perpendicular magnetic anisotropy in $\mathrm{Mo} / \mathrm{CoFeB} / \mathrm{MgO}$ magnetic tunnel junctions. Appl. Phys. Lett. 106, 182406 (2015).

23. Cho, S., Baek, S.-H. C., Lee, K.-D., Jo, Y. \& Park, B.-G. Large spin Hall magnetoresistance and its correlation to the spin-orbit torque in $\mathrm{W} / \mathrm{CoFeB} / \mathrm{MgO}$ structures. Sci. Rep. 5, 14668 (2015).

24. Pai, C. F. et al. Spin transfer torque devices utilizing the giant spin Hall effect of tungsten. Appl. Phys. Lett. 101, 122404 (2012).

25. Liu, J., Ohkubo, T., Mitani, S., Hono, K. \& Hayashi, M. Correlation between the spin Hall angle and the structural phases of early $5 d$ transition metals. Appl. Phys. Lett. 107, 232408 (2015).

26. He, C. et al. Spin-torque ferromagnetic resonance measurements utilizing spin Hall magnetoresistance in $\mathrm{W} / \mathrm{Co}_{40} \mathrm{Fe}_{40} \mathrm{~B}_{20} / \mathrm{MgO}$ structures. Appl. Phys. Lett. 109, 202404 (2016).

27. Skowroński, W. et al. Underlayer material influence on electric-field controlled perpendicular magnetic anisotropy in $\mathrm{CoFeB} / \mathrm{MgO}$ magnetic tunnel junctions. Phys. Rev. B 91, 184410 (2015).

28. Chatterjee, J. et al. Enhanced annealing stability and perpendicular magnetic anisotropy in perpendicular magnetic tunnel junctions using W layer. Appl. Phys. Lett. 110, 202401 (2017).

29. van Kampen, M. et al. All-optical probe of coherent spin waves. Phys. Rev. Lett. 88, 227201 (2002).

30. Mizukami, S. et al. Laser-induced fast magnetization precession and Gilbert damping for $\mathrm{CoCrPt}$ alloy thin films with perpendicular magnetic anisotropy. Appl. Phys. Express 3, 123001 (2010).

31. Mizukami, S. et al. Long-lived ultrafast spin precession in manganese alloys films with a large perpendicular magnetic anisotropy. Phys. Rev. Lett. 106, 117201 (2011).

32. Iihama, S. et al. Observation of precessional magnetization dynamics in $\mathrm{L} 1_{0}-\mathrm{FePt}$ thin films with different $\mathrm{L}_{0}$ order parameter values. Jpn. J. Appl. Phys. 52, 073002 (2013).

33. Zhu, J., Wu, X., Lattery, D. M., Zheng, W. \& Wang, X. The ultrafast laser pump-probe technique for thermal characterization of materials with micro/nanostructures. Nanosc. Microsc. Therm. 21, 177-198 (2017).

34. Takahashi, Y. K. et al. Increased magnetic damping in ultrathin films of $\mathrm{Co}_{2} \mathrm{FeAl}$ with perpendicular anisotropy. Appl. Phys. Lett. 110, 252409 (2017).

35. You, C. Y. \& Shin, S. C. Generalized analytic formulae for magneto-optical Kerr effects. J. Appl. Phys. 84, 541-546 (1998).

36. Krivosik, P., Mo, N., Kalarickal, S. \& Patton, C. E. Hamiltonian formalism for two magnon scattering microwave relaxation: Theory and applications. J. Appl. Phys. 101, 083901 (2007).

37. Iihama, S. et al. Gilbert damping constants of $\mathrm{Ta} / \mathrm{CoFeB} / \mathrm{MgO}(\mathrm{Ta})$ thin films measured by optical detection of precessional magnetization dynamics. Phys. Rev. B 89, 174416 (2014).

38. Kittel, C. On the theory of ferromagnetic resonance absorption. Phys. Rev. 73, 155-161 (1948)

39. Ma, L. et al. Tunable magnetization dynamics in disordered FePdPt ternary alloys: Effects of spin orbit coupling. J. Appl. Phys. 116, 113908 (2014).

40. Mizukami, S. Fast magnetization precession and damping for magnetic films with high perpendicular magnetic anisotropy. J. Magn. Soc. Jpn. 39, 1-7 (2015).

41. Suhl, H. Ferromagnetic resonance in nickel ferrite between one and two kilomegacycles. Phys. Rev. 97, 555-557 (1955).

42. Wang, D. S. et al. High thermal stability and low Gilbert damping constant of $\mathrm{CoFeB} / \mathrm{MgO}$ bilayer with perpendicular magnetic anisotropy by Al capping and rapid thermal annealing. Appl. Phys. Lett. 104, 142402 (2014).

43. Capua, A., Yang, S. H., Phung, T. \& Parkin, S. S. P. Determination of intrinsic damping of perpendicularly magnetized ultrathin films from time-resolved precessional magnetization measurements. Phys. Rev. B 92, 9 (2015).

44. An, G.-G. et al. Highly stable perpendicular magnetic anisotropies of $\mathrm{CoFeB} / \mathrm{MgO}$ frames employing $\mathrm{W}$ buffer and capping layers. Acta Mater. 87, 259-265 (2015).

45. Yilgin, R., Oogane, M., Yakata, S., Ando, Y. \& Miyazaki, T. Intrinsic Gilbert damping constant in $\mathrm{Co}_{2} \mathrm{MnAl}_{\mathrm{Ae}} \mathrm{Heu}$ ler alloy films. IEEE Trans. Magn. 41, 2799-2801 (2005).

46. Iihama, S. et al. Low precessional damping observed for $\mathrm{L}_{0}$-ordered FePd epitaxial thin films with large perpendicular magnetic anisotropy. Appl. Phys. Lett. 105, 142403 (2014).

47. Skowroński, W. et al. Temperature dependence of spin-orbit torques in W/CoFeB bilayers. Appl. Phys. Lett. 109, 062407 (2016).

\section{Acknowledgements}

This work is supported by C-SPIN (award \#: 2013-MA-2381), one of six centers of STARnet, a Semiconductor Research Corporation program, sponsored by MARCO and DARPA. X.H. thanks Niron Magnetics, Inc. for financial support. The sample structural characterizations (XRD and XRR) were performed at the University of Minnesota Characterization Facility (CharFac), which received capital equipment funding from the University of Minnesota MRSEC under NSF Award DMR-1420013. The authors would like to thank Prof. Paul Crowell and Dr. Changjiang Liu for valuable discussions.

\section{Author Contributions}

D.Z. and D.L. conceived the idea and designed the measurements, under the supervision of X.W. and J.W., D.Z. synthesized the CoFeB samples and conducted VSM measurements. X.H. performed all the XRD and XRR characterizations. D.L. carried out the TR-MOKE measurements and led the manuscript writing with a portion of the materials provided by D.Z., J.Z., D.L. and D.Z. contributed to analyzing the data and revising the manuscript. All authors have reviewed and discussed the results and conclusions of this work. 


\section{Additional Information}

Supplementary information accompanies this paper at https://doi.org/10.1038/s41598-018-31642-9.

Competing Interests: The authors declare no competing interests.

Publisher's note: Springer Nature remains neutral with regard to jurisdictional claims in published maps and institutional affiliations.

(c) (1) Open Access This article is licensed under a Creative Commons Attribution 4.0 International License, which permits use, sharing, adaptation, distribution and reproduction in any medium or format, as long as you give appropriate credit to the original author(s) and the source, provide a link to the Creative Commons license, and indicate if changes were made. The images or other third party material in this article are included in the article's Creative Commons license, unless indicated otherwise in a credit line to the material. If material is not included in the article's Creative Commons license and your intended use is not permitted by statutory regulation or exceeds the permitted use, you will need to obtain permission directly from the copyright holder. To view a copy of this license, visit http://creativecommons.org/licenses/by/4.0/.

(C) The Author(s) 2018 